International Journal of Modern Physics A

Vol. 29, No. 19 (2014) 1443009 (17 pages)

(C) World Scientific Publishing Company

DOI: $10.1142 / S 0217751 X 1443009 X$

\title{
Scintillating bolometers: A key for determining WIMP parameters
}

\author{
D. G. Cerdeño, C. Marcos and M. Peiró \\ Instituto de Física Teórica UAM/CSIC, \\ Universidad Autónoma de Madrid, 28049 Madrid, Spain \\ Departamento de Física Teórica, \\ Universidad Autónoma de Madrid, 28049 Madrid, Spain
}

\section{Fornasa}

School of Physics and Astronomy, University of Nottingham, University Park, Nottingham NGr 2RD, UK

C. Cuesta, ${ }^{*}$ E. García, C. Ginestra, M. Martínez, ${ }^{\dagger}$ Y. Ortigoza,

J. Puimedón and M. L. Sarsa

Grupo de Física Nuclear y Astropartículas, Universidad de Zaragoza, 50009 Zaragoza, Spain

Laboratorio Subterráneo de Canfranc, Paseo de los Ayerbe s.n., 22880 Canfranc Estación, Huesca, Spain

${ }^{\dagger}$ mariam@unizar.es

\author{
Received 14 January 2014 \\ Accepted 15 March 2014 \\ Published 15 July 2014
}

\begin{abstract}
In the last decade direct detection Dark Matter (DM) experiments have increased enormously their sensitivity and ton-scale setups have been proposed, especially using germanium and xenon targets with double readout and background discrimination capabilities. In light of this situation, we study the prospects for determining the parameters of Weakly Interacting Massive Particle (WIMP) DM (mass, spin-dependent (SD) and spin-independent (SI) cross-section off nucleons) by combining the results of such experiments in the case of a hypothetical detection. In general, the degeneracy between the SD and SI components of the scattering cross-section can only be removed using targets with different sensitivities to these components. Scintillating bolometers, with particle discrimination capability, very good energy resolution and threshold and a wide choice of target materials, are an excellent tool for a multitarget complementary DM search. We investigate how the simultaneous use of scintillating targets with different SD-SI sensitivities and/or light isotopes (as the case of $\mathrm{CaF}_{2}$ and $\mathrm{NaI}$ ) significantly improves the
\end{abstract}

\footnotetext{
* Present address: CENPA, University of Washington, USA.

†Fundación ARAID, C/María de Luna 11, Edificio CEEI Aragón, 50018 Zaragoza, Spain.
} 


\begin{abstract}
determination of the WIMP parameters. In order to make the analysis more realistic we include the effect of uncertainties in the halo model and in the spin-dependent nuclear structure functions, as well as the effect of a thermal quenching different from 1.
\end{abstract}

Keywords: Dark matter; direct detection; complementarity; bolometers.

PACS numbers: 95.35.+d, 29.40.Vj

\title{
1. Introduction
}

Weakly Interacting Massive Particles (WIMPs) can be directly detected through their scattering off target nuclei of a detector. ${ }^{1}$ In the last decades, numerous experiments, using different targets and detection techniques, have been searching for WIMPs or are currently taking data. Some of them have searched for distinctive signals, such as an annual modulation in the detection rate: $\mathrm{DAMA}^{2}$ and DAMA/LIBRA, ${ }^{3,4}$ using NaI scintillators, have reported a highly significant signal $(9.3 \sigma)$ and CoGeNT ${ }^{5,6}$ claimed a less significant evidence $(2.2 \sigma)$ in the first three years of its data, gathered with a Ge semiconductor. Moreover, CoGeNT, ${ }^{7}$ $\mathrm{CRESST}^{8}$ (using $\mathrm{CaWO}_{4}$ scintillating bolometers) and CDMS II (with data from its $\mathrm{Si}$ detectors $)^{9}$ have reported excesses of events at low energies that could be compatible with a signal produced by light WIMPs with a mass of the order of $10 \mathrm{GeV}$. On the other hand, XENON10, ${ }^{10}$ XENON100, ${ }^{11} \mathrm{LUX}^{12}$ (also based on Xe), the abovementioned CDMS II, ${ }^{13}$ EDELWEISS ${ }^{14,15}$ (with Ge), KIMS $^{16}$ (with CsI), PICASSO ${ }^{17}$ (with $\mathrm{C}_{4} \mathrm{~F}_{10}$ ), SIMPLE $^{18}$ (with $\mathrm{C}_{2} \mathrm{ClF}_{5}$ ) and COUPP ${ }^{19}$ (with $\mathrm{CF}_{3} \mathrm{I}$ ) have obtained negative results setting more stringent upper bounds on the WIMP-nucleon cross-sections. Currently the strongest limits are obtained by the LUX collaboration, excluding spin-independent WIMP-nucleon elastic scattering cross-sections larger than $7.6 \times 10^{-46} \mathrm{~cm}^{2}$ for a WIMP mass of $33 \mathrm{GeV}$, and the SuperCDMS collaboration for low mass WIMPs. ${ }^{20,21}$ In the next years new experiments and upgraded versions of the existing ones are going to explore even smaller cross-sections, closing in on DM searches.

The final goal of all these experiments is to determine the nature of DM, measuring some of its properties (namely its mass and interaction cross-section with ordinary matter). Signals from different targets are needed, since they can provide complementary information which can lead to a better determination of the DM parameters. ${ }^{22,23}$ In a previous paper ${ }^{24}$ we analyzed the complementarity of a Ge and a Xe experiment with energy thresholds and resolutions already achieved by CDMS and XENON100 experiments, respectively, and with background levels expected for their corresponding extensions (SuperCDMS ${ }^{25}$ and XENON1T ${ }^{26}$ ). For different WIMP scenarios, we assumed hypothetical detections with an exposure of $300 \mathrm{~kg} \times \mathrm{yr}$ in both experiments and we concluded that the combination of data from Xe- and Ge-based detectors might not lead to a good reconstruction of all the WIMP parameters, since there is a degeneracy in the SI and SD parts of the scattering WIMP-nucleus cross-section, and both targets have very similar SI over SD sensitivity. We showed that incorporating targets with different sensitivities to SI 
and SD interactions could significantly improve the reconstruction. We considered the case of some of the most promising scintillating bolometric targets: $\mathrm{CaWO}_{4}$ (currently used by CRESST), $\mathrm{Al}_{2} \mathrm{O}_{3}$ and $\mathrm{LiF}$ (studied by ROSEBUD, ${ }^{27}$ that could be considered in the future as additional targets in EURECA, ${ }^{28}$ a European collaboration that plans to search for WIMPs with a 1-ton cryogenic hybrid detector).

We observed that the inclusion of $\mathrm{CaWO}_{4}$ (being mainly sensitive to SI couplings) only leads to a total complementary result for a WIMP of $50 \mathrm{GeV}$ in a small region of the plane $\left(\sigma^{\mathrm{SI}}, \sigma^{\mathrm{SD}}\right)$ in which the expected events in Ge and Xe are mainly due to SD interactions. On the other hand, $\mathrm{Al}_{2} \mathrm{O}_{3}$ and $\mathrm{LiF}$ (being more sensitive to SD interactions) achieve complementarity with germanium and xenon in regions of the parameter space where the rate in the latter is dominated by SI couplings. We also determined the exposures and background levels required by the bolometers to be complementary to Ge- and Xe-based experiments.

In this paper we follow the same strategy and reanalyze the role of Ge- and Xe-based experiments in light of the improved (or potential) energy thresholds in CDMS and LUX. ${ }^{a}$ We also study the complementarity with two additional targets: $\mathrm{CaF}_{2}$ and NaI. The first one has already been used as scintillating bolometer, ${ }^{30,31}$ whereas the construction of a bolometer based on NaI (which is a hygroscopic and fragile material) is an ongoing R\&D project of the Zaragoza group. ${ }^{32}$ We include in our analysis not only the effect of the previously considered uncertainties in the halo parameters and SD structure functions, but also the possible influence of the thermal quenching between nuclear and electron recoils in the complementarity of these targets.

The structure of this article is as follows. Section 2 is a short summary of the methodology we follow in reconstructing the WIMP parameters from the (simulated) data in direct detection experiments. In Subsec. 2.1 we address the most relevant uncertainties in the analysis, in particular the astrophysical ones (due to our imperfect knowledge of the DM halo of the Milky Way), those related to the SD Structure Functions (SDSF) parametrizing the spin content of the nucleons in the target and, finally, the effect of changing the thermal quenching $q$. In Subsec. 2.2 we present the results for some selected benchmarks when considering only Ge and Xe experiments, finding that the combination of data from these two targets contributes to a better measurement of the WIMP parameters, but a degeneracy in the SD and SI independent cross-section usually remains. In Sec. 3 we describe the characteristics of the scintillating targets under study (i.e. $\mathrm{CaF}_{2}$ and $\mathrm{NaI}$ ). In Sec. 4 we show how their inclusion can lead to a better determination of the DM mass and scattering cross-section, breaking in some cases the SI-SD degeneracy. Finally, conclusions are presented in Sec. 5 .

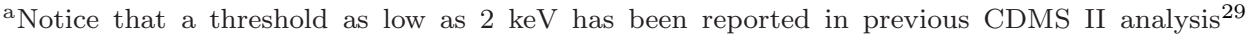
although not for a background free search. In order to simplify the comparison with LUX, we will here assume the same threshold of $3 \mathrm{keV}$, considering that the new iZIP detectors in SuperCDMS might allow a much better background subtraction.
} 


\section{Reconstructing WIMP Parameters from Signals in Direct Detection Experiments}

In the standard analysis framework for WIMP direct detection ${ }^{33,34}$ (see also Refs. 35 and 36 for recent reviews) the WIMP-nucleus scattering cross-section is separated into a SI and a SD contribution, with $f_{p}$ and $a_{p}$ being the corresponding effective couplings to protons and $f_{n}$ and $a_{n}$ to neutrons. In order to reduce the number of parameters that characterize the expected event rate, we assume here that the SI coupling is isospin-invariant $\left(f_{p}=f_{n}\right)$ and we take a specific relation between $a_{p}$ and $a_{n}$ (namely $a_{p} / a_{n}=-1$ ). Under these assumptions, the generic WIMP is completely determined by its mass $m_{\chi}$, the SI contribution to the WIMP-nucleon cross-section $\sigma^{\mathrm{SI}}$ and the SD component $\sigma^{\mathrm{SD}}$.

Thus, the total number of WIMP recoil events in a given energy window can be expressed as

$$
N=\sum_{\text {isotopes }} f\left(\mathcal{C}_{\mathrm{SI}} \sigma^{\mathrm{SI}}+\mathcal{C}_{\mathrm{SD}} 4\left(S_{p}-S_{n}\right)^{2} \sigma^{\mathrm{SD}}\right)
$$

where, for each isotope, $f$ is its mass fraction in the detector, $S_{p}$ and $S_{n}$ are the expectation values of the total spin operators for protons and neutrons respectively and the coefficients $\mathcal{C}_{\mathrm{SI}}$ and $\mathcal{C}_{\mathrm{SD}}$ can be written as follows:

$$
\begin{aligned}
\mathcal{C}_{\mathrm{SI}} & \equiv \int d E_{R} \int\left(\frac{\epsilon \rho_{0} f(v)}{2 \mu_{n}^{2} m_{\chi} v}\right) A^{2} F_{\mathrm{SI}}^{2} d v \\
\mathcal{C}_{\mathrm{SD}} & \equiv \int d E_{R} \int\left(\frac{\epsilon \rho_{0} f(v)}{2 \mu_{n}^{2} m_{\chi} v}\right)\left(\frac{J+1}{3 J}\right) F_{\mathrm{SD}}^{2} d v .
\end{aligned}
$$

$\epsilon$ is the experimental exposure, $\rho_{0}$ is the local WIMP density, $f(v)$ is the WIMP speed distribution in the Earth reference frame normalized to unity, $\mu_{n}$ is the WIMP-nucleon reduced mass, $E_{R}$ is the nucleus recoil energy, $F_{\mathrm{SI}}^{2}\left(F_{\mathrm{SD}}^{2}\right)$ is the $\mathrm{SI}(\mathrm{SD})$ nuclear form factor, $A$ is the nucleus mass number, and $J$ its nuclear spin.

We focus the analysis on two benchmark cases. For each of them (and for each target independently) we calculate the signal that such WIMPs would produce in that specific detector by computing the number of recoil events, $\left\{\lambda_{i}\right\}^{a}$, expected for target $a$ in the $i$ th bin of $N$ evenly-spaced energy bins contained in the energy window for WIMP search of each experiment. These expected events, $\left\{\lambda_{i}\right\}^{a}$, represent our experimental data, $\mathbf{D}$, and we are interested in estimating how well such simulated measurements can be used to reconstruct the WIMP parameters.

In order to do so, we perform scans over the parameter space $\left(m_{\chi}=1-10^{5} \mathrm{GeV}\right.$, $\sigma^{\mathrm{SI}}=10^{-12}-10^{-6} \mathrm{pb}$, and $\left.\sigma^{\mathrm{SD}}=10^{-8}-1 \mathrm{pb}\right)$. For every point in the scan we compute the number of recoil events $N_{i}^{a}$ in the $i$ th energy bin for every target $a$ and then compute the likelihood comparing $N_{i}^{a}$ with the prediction of the benchmark model in the same energy bin for the same target, assuming that data from each experiment follow independent Poissonian distributions. We present the results as $68 \%$ and $99 \%$ confidence regions in the profile likelihood (PL). The nuclear and astrophysical uncertainties are considered as nuisance parameters. The scans are 
performed with MultiNest $3.0^{37}$ interfaced with our own code for the computation of the number of recoil events and of the likelihood. Logarithmic flat priors are assumed for the three variables. We refer to Ref. 24 for a detailed description of how the scans are performed.

If only one target is considered, the reconstruction of WIMP parameters is affected by degeneracies, since the number of events detected can be explained by different combinations of $\left(m_{\chi}, \sigma^{\mathrm{SI}}, \sigma^{\mathrm{SD}}\right)$. Such degeneracy can be broken by including more targets in the analysis: in Ref. 24 we defined "complementarity" as the situation in which a certain set of experiments manages to determine $m_{\chi}$, $\sigma^{\mathrm{SI}}$ and $\sigma^{\mathrm{SD}}$ with a certain finite accuracy, or, equivalently, when $68 \%$ confidence level of the two-dimensional contours are closed simultaneously in the three planes $\left(m_{\chi}, \sigma^{\mathrm{SI}}\right),\left(m_{\chi}, \sigma^{\mathrm{SD}}\right)$ and $\left(\sigma^{\mathrm{SI}}, \sigma^{\mathrm{SD}}\right)$.

The following two WIMP benchmarks will be considered in the remaining sections:

- VL-SI: $m_{\chi}=20 \mathrm{GeV}, \sigma^{\mathrm{SI}}=10^{-9} \mathrm{pb}, \sigma^{\mathrm{SD}}=10^{-5} \mathrm{pb}$, corresponding to a very light WIMP for which the SI contribution dominates the detection rate in Ge and Xe,

- L-SD: $m_{\chi}=50 \mathrm{GeV}, \sigma^{\mathrm{SI}}=10^{-10} \mathrm{pb}, \sigma^{\mathrm{SD}}=1.5 \times 10^{-4} \mathrm{pb}$, a light WIMP for which the SD contribution dominates in Ge and Xe.

\subsection{Including uncertainties}

The expected DM signal depends on parameters affected by large uncertainties. In the following, we will take into account uncertainties in the velocity distribution of DM in the Milky Way halo, the spin dependent form functions for the target nuclei and the performance of the detector.

We considered a velocity distribution function that differs from the standard halo model by the presence of a high-velocity tail. Such a model, adopted from Ref. 38 , is well motivated by $N$-body simulations and the velocity distribution can be written as follows,

$$
F(v)=N_{k}^{-1} v^{2}\left[e^{-v^{2} / k v_{0}^{2}}-e^{-v_{\text {esc }}^{2} / k v_{0}^{2}}\right]^{k} \Theta\left(v_{\text {esc }}-v\right),
$$

where $N_{k}=v_{0}^{3} e^{-y_{e}^{2}} \int_{0}^{y_{e}} y^{2}\left(e^{-\left(y^{2}-y_{e}^{2}\right) / k}-1\right)^{k} d y, y_{e}=v_{\text {esc }} / v_{0}$ and $k$ is the parameter that quantifies the deviation from the standard halo model, recovering it in the limiting case of $k=0 .{ }^{38}$ This expression for the velocity distribution depends on three parameters: $v_{\mathrm{esc}}, v_{0}$ and $k$. In order to account for our ignorance on the true velocity distribution of the DM in the halo of our Galaxy we leave such parameters free to vary within the following ranges: $v_{\mathrm{esc}}=[478,610] \mathrm{km} / \mathrm{s}, v_{0}=[170,290] \mathrm{km} / \mathrm{s}$, and $k=[0.5,3.5]$. We also scan over the local WIMP density $\rho_{0}$, in the range between 0.2 and $0.6 \mathrm{GeV} / \mathrm{cm}^{3}$. All these parameters are subject to a uniformly flat prior distribution. 
Table 1. Ranges considered for the parameters $N, \alpha$ and $\beta$ that describe the SDSFs (see Eq. (4)) for the isotopes studied in this work.

\begin{tabular}{lccc}
\hline Isotope & $N$ & $\alpha$ & $\beta$ \\
\hline${ }^{73} \mathrm{Ge}$ & $0.0749-0.2071$ & $5.0-6.0$ & $0.0304-0.0442$ \\
${ }^{129} \mathrm{Xe}$ & $0.0225-0.0524$ & $4.0625-4.3159$ & $0.001-0.0093$ \\
${ }^{131} \mathrm{Xe}$ & $0.0169-0.0274$ & $3.9913-4.7075$ & $0.05-0.105$ \\
${ }^{127} \mathrm{I}$ & $0.0297-0.0568$ & $4.0050-4.4674$ & $0.05-0.057$ \\
${ }^{23} \mathrm{Na}$ & $0.0098-0.0277$ & $2.0-3.5287$ & $0-0.1250$ \\
${ }^{19} \mathrm{~F}$ & $0.0505-0.1103$ & $2.9679-3.0302$ & $0-0.0094$ \\
\hline
\end{tabular}

Regarding the WIMP interaction with the nucleus, it has been shown in Ref. 39 that uncertainties in the SI and SD form factors of the target nuclei play a very different role. In the case of SI interactions, differences in the form factor can be safely neglected (in the present paper we have used the Helm form factor). On the other hand, for SD interactions the expressions of the form factors are more dependent on the nuclear model. These differences can significantly affect the expected WIMP rate and, thus, the reconstruction of the WIMP parameters (especially when SD interactions play a relevant role).

To take into account such uncertainties, the SDSFs are parametrized as follows, ${ }^{39}$

$$
S_{i j}(u)=N\left((1-\beta) e^{-\alpha u}+\beta\right),
$$

where $u$ is an adimensional quantity proportional to the square of the momentum transfer, $u=(q b)^{2} / 2$, in terms of the oscillator size parameter $b=A^{1 / 6}$. Note that for the case $a_{p} / a_{n}=-1$ the only contribution comes from the $S_{11}$ SDSF. Table 1 shows the ranges in which the three parameters $N, \alpha$ and $\beta$ have been allowed to vary for each nucleus in order to reproduce the various determinations of the form factors available in the literature (see Ref. 39 for a detailed explanation). Results for the SDSFs of the isotopes relevant in this work in the case $a_{p} / a_{n}=-1$ are displayed in Fig. 1 (light blue area) together with the most relevant nuclear calculations.

Finally, important systematics can also arise from the detection technique itself. Among these, we consider the effect of the thermal quenching factor, $q$, that measures the relative efficiency in the conversion into measurable thermal signal of the nuclear recoils energy deposition with respect to that corresponding to electron recoils, since the detectors are calibrated with gamma sources and the measured spectra are given in electron-equivalent energy. This factor is typically assumed equal to one for bolometers but small deviations (of about 10-15\%) have been measured in different detectors (see for example Ref. 46 and references therein). To illustrate the influence of this uncertainty on $q$, we consider here three different values $(q=0.85,1$, and 1.15$)$ for the NaI target. 


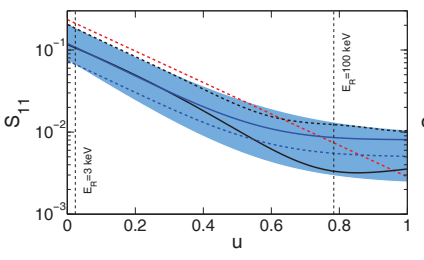

(a) ${ }^{73} \mathrm{Ge}$

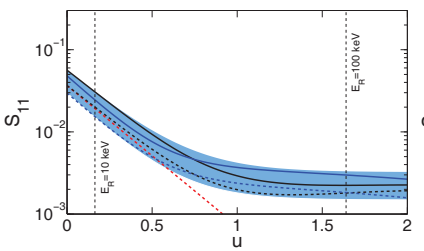

(d) ${ }^{127} \mathrm{I}$

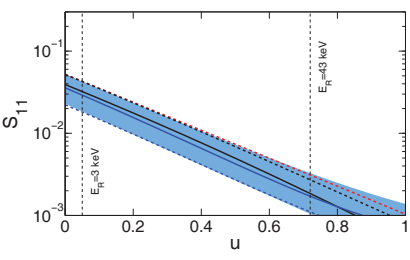

(b) ${ }^{129} \mathrm{Xe}$

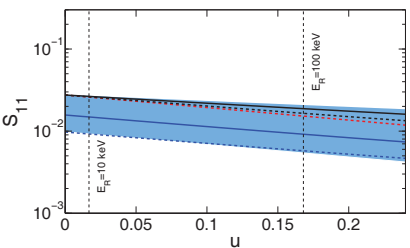

(e) ${ }^{23} \mathrm{Na}$

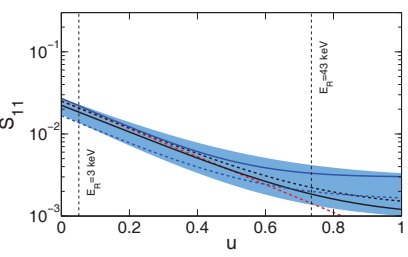

(c) ${ }^{131} \mathrm{Xe}$

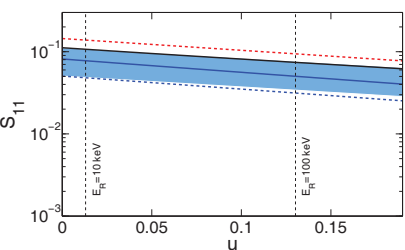

(f) ${ }^{19} \mathrm{~F}$

Fig. 1. (Color online) Light blue area: SDSFs as a function of $u=\left(q A^{1 / 6}\right)^{2} / 2$ for $a_{p} / a_{n}=-1$ from Eq. (4) (parameters varying within the ranges of Table 1). Blue dashed (solid) line: Klos et al. min (max) model. ${ }^{40}$ Red dashed line: Gaussian approximation. ${ }^{41}$ (a) Black dashed (solid) line: Resell et al. ${ }^{42}$ (Dimitrov et al. ${ }^{43}$ ). (b)-(d) Black dashed (solid) line: Bonn (Nijm). ${ }^{44}$ (e) Black dashed (solid) line: Resell-Dean ${ }^{44}$ (Vergados et al. ${ }^{45}$ ). (f) Black solid line: Vergados et al. ${ }^{45}$

\subsection{Results for Ge and $\mathrm{Xe}$}

As in our previous paper, ${ }^{24}$ we start the analysis studying the complementarity of two experiments, based, respectively on Ge and Xe. Such elements are employed by the collaborations producing the currently most stringent limits on WIMP properties and are contemplated in projects planning to extend the search to the ton scale (e.g. EURECA, SuperCDMS and XENON1T) or even to the multi-ton scale (LZ ${ }^{47}$ and DARWIN ${ }^{48}$ ). Consequently, these targets are expected to represent the most sensitive experiments (at least in the most general WIMP scenarios) in the near future.

For our study, we have assumed a positive result (WIMP detection) in two experiments, one using a Ge-based target and the other using Xe. We consider the two detections combined when reconstructing the WIMP parameters. The same exposure $(\epsilon=300 \mathrm{~kg} \times \mathrm{yr})$ is assumed for both experiments, as well as zero background. ${ }^{\mathrm{b}}$ The energy window is set to [3-100] keV for Ge and [3-43] keV for Xe, where the lower values account for the recent or potential improvements in nuclear recoil energy thresholds of some Xe and Ge experiments. Table 2 shows the expected number of WIMP recoil events for the considered benchmarks over the whole energy range.

${ }^{\mathrm{b}}$ In our previous work $^{24}$ we checked that the expected backgrounds for SuperCDMS and XENON1T are so low that have no impact in the results, so zero background can be safely assumed. 
Table 2. Benchmark points used: VL-SI is a very light WIMP with dominant SI scattering cross-section while L-SD has a significant SD contribution (L-SD). The fifth and sixth columns indicate the total expected recoil events in Ge- and Xe-based experiments for an exposure $\epsilon=300 \mathrm{~kg} \times \mathrm{yr}$, in the energy windows [3-100] keV for $\mathrm{Ge}$ and [3-43] keV for Xe. The number in parenthesis indicates the expected recoils when only SI interactions are considered. In the calculation we considered the mean values of the parameters describing the SDSFs (see Table 1) and the halo model of Eq. (3) with $\rho_{0}=0.4 \mathrm{GeV} / \mathrm{cm}^{3}, v_{0}=220 \mathrm{~km} / \mathrm{s}, v_{\text {esc }}=544 \mathrm{~km} / \mathrm{s}$ and $k=2$.

\begin{tabular}{lccccc}
\hline & $m_{\chi}(\mathrm{GeV})$ & $\sigma^{\mathrm{SI}}(\mathrm{pb})$ & $\sigma^{\mathrm{SD}}(\mathrm{pb})$ & $N_{\mathrm{Ge}}$ & $N_{\mathrm{Xe}}$ \\
\hline VL-SI & 20 & $10^{-9}$ & $10^{-5}$ & $40.4(39.3)$ & $65.0(61.6)$ \\
L-SD & 50 & $10^{-10}$ & $1.5 \times 10^{-4}$ & $29.3(6.1)$ & $94.7(11.0)$ \\
\hline
\end{tabular}
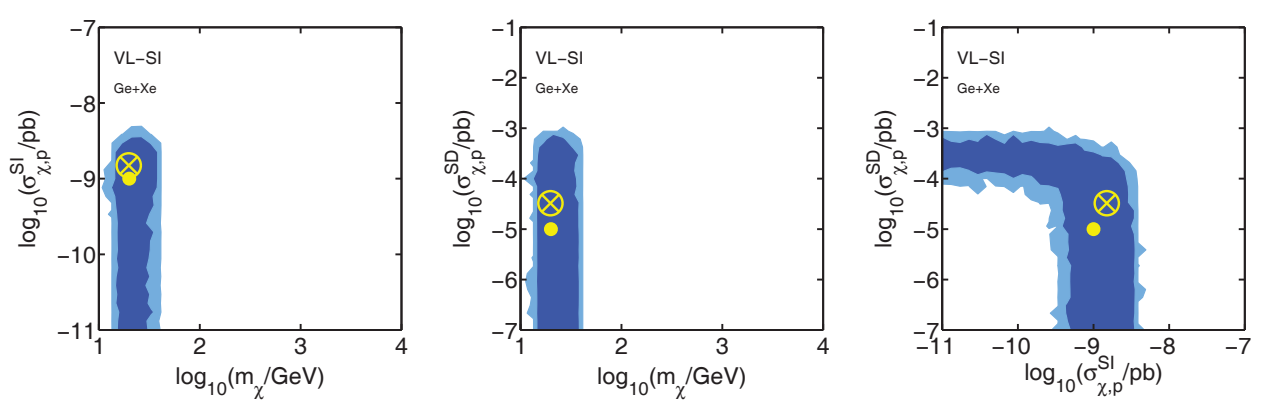

Fig. 2. (Color online) Profile likelihood contours at 68 and 99\% C.L. for Ge+Xe. All uncertainties have been included in the analysis. The yellow dot denotes the benchmark VL-SI and the circled cross the best fit point.

Figure 2 shows the $68 \%$ and $99 \%$ confidence level contours for the three WIMP parameters projected onto the corresponding two-dimensional plots $\left(\sigma^{\mathrm{SI}}, m_{\chi}\right)$, $\left(\sigma^{\mathrm{SD}}, m_{\chi}\right)$, and $\left(\sigma^{\mathrm{SD}}, \sigma^{\mathrm{SI}}\right)$ for the benchmark VL-SI. The yellow dot represents the nominal value and the circled cross is the best-fit point. As we showed in Ref. 24, the combination of data from Ge and Xe leads to a substantial reduction in the contours of the reconstructed WIMP parameters. The improved energy threshold also contributes to this. In particular, for this benchmark the mass of the WIMP can be well determined (the contours using only one target would not be closed). However, there remains a degeneracy in both cross-sections, $\sigma^{\mathrm{SI}}$ and $\sigma^{\mathrm{SD}}$, for which only upper limits are derived. This is due to the similar sensitivity to SI/SD interactions of $\mathrm{Ge}$ and $\mathrm{Xe}$ in this point.

Analogously, Fig. 3 displays the contour plots for the benchmark L-SD. The reconstruction of WIMP parameters is similar, although in this case $\sigma^{\mathrm{SD}}$ is better bounded and even a lower limit is derived at $68 \%$ C.L. Nevertheless, at 99\% C.L. the degeneracy between $\sigma^{\mathrm{SI}}$ and $\sigma^{\mathrm{SD}}$ still remains and only an upper bound is obtained for $\sigma^{\mathrm{SI}}$ which is far from the nominal value.

Thus, although the combined data from Ge and Xe experiments can be used to significantly improve the determination of WIMP parameters, the degeneracy in the 

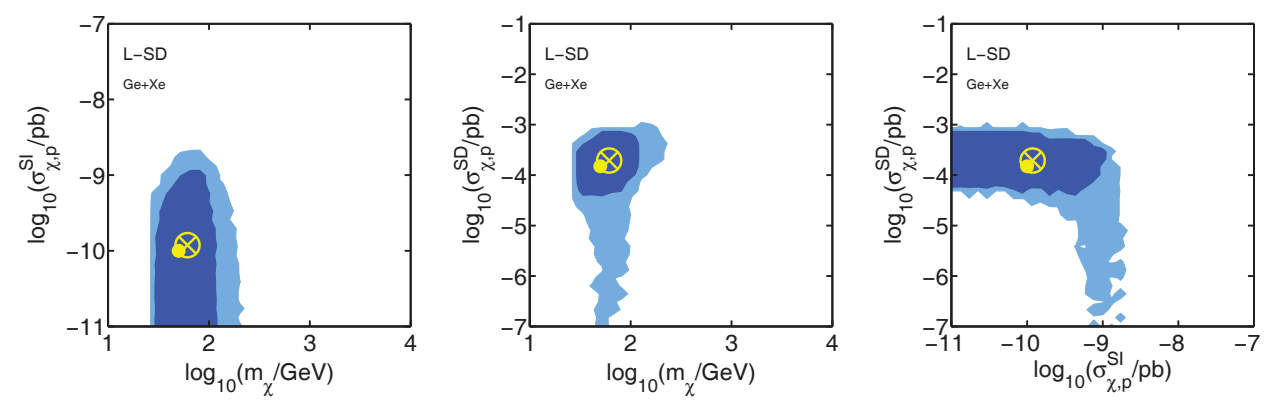

Fig. 3. (Color online) The same as Fig. 2 but for the L-SD benchmark.

SI and SD components of the scattering cross-section might be difficult to break. As we will argue in the following sections, incorporating data from a third target with a different sensitivity to these components can help solving this problem.

\section{Scintillating Bolometers for Dark Matter Searches}

Compared to other hybrid detectors with discrimination capability, scintillating bolometers have the advantage of a wide target choice. This makes it possible to select intrinsically radiopure materials and combine different nuclei to maximize the explored region of the WIMP parameter space (high mass number $A$ for large SI coupling, low $A$ to enhance sensitivity to light WIMPs, or nonzero nuclear spin for sensitivity to SD interaction, to name just a few possibilities).

The energy threshold that has been achieved in the heat signal with cryogenic detectors is as low as $\sim 1 \mathrm{keV}$. However, when looking for nuclear recoils, the discrimination threshold is determined by the target light yield and the sensitivity of the optical detector. Usually this role is played by a second low-mass large-area bolometer facing the primary one. Optimizing the sensitivity and response of the optical bolometer is a very active ongoing research field (see for example Refs. 49 and 50) and lower thresholds are expected in a near future. Nevertheless, in this paper, we follow the same approach of Ref. 24 and take a reference energy threshold of $10 \mathrm{keV}$ (a value already observed in some scintillating bolometers ${ }^{8,51}$ ) for the bolometric targets under study.

In our previous work ${ }^{24}$ we studied the complementarity of three scintillating materials: $\mathrm{CaWO}_{4}, \mathrm{Al}_{2} \mathrm{O}_{3}$ and $\mathrm{LiF}$. $\mathrm{CaWO}_{4}$ is the current target of the CRESST experiment ${ }^{8}$ and was used also by the ROSEBUD collaboration in the first underground DM search with light and heat discrimination. ${ }^{52}$ It constitutes the baseline for the EURECA scintillating targets. $\mathrm{Al}_{2} \mathrm{O}_{3}$, used by ROSEBUD ${ }^{53,54}$ and by CRESST in the first phase of the experiment, ${ }^{55}$ is particularly interesting for its sensitivity to low mass WIMPs. Finally, LiF, also sensitive to light WIMPs and SD interaction, has been used by the ROSEBUD collaboration for DM searches and as neutron detector, showing that its use in a DM experiment could allow for thermal neutron monitoring. ${ }^{56,57}$ However, the light yields achieved so far do not provide a 
Table 3. Number of WIMP recoils expected in the bolometric targets for the benchmarks (BM) described in Sec. 2. In both cases data correspond to an exposure of $\epsilon=300 \mathrm{~kg} \times \mathrm{yr}$ and [10-100] keV energy window. The number in parenthesis indicate the contribution from SI interaction. For NaI three different values of the quenching factor have been considered.

\begin{tabular}{lcccccrr}
\hline & $\begin{array}{c}m_{\chi} \\
(\mathrm{GeV})\end{array}$ & $\begin{array}{c}\sigma^{\mathrm{SI}} \\
(\mathrm{pb})\end{array}$ & $\begin{array}{c}\sigma^{\mathrm{SD}} \\
(\mathrm{pb})\end{array}$ & $\begin{array}{c}N_{\mathrm{NaI}} \\
q=0.85\end{array}$ & $\begin{array}{c}N_{\mathrm{NaI}} \\
q=1\end{array}$ & $\begin{array}{c}N_{\mathrm{NaI}} \\
q=1.15\end{array}$ & \multicolumn{1}{c}{$\begin{array}{c}N_{\mathrm{CaF}_{2}} \\
q=1\end{array}$} \\
\hline VL-SI & 20 & $10^{-9}$ & $10^{-5}$ & $3.5(2.9)$ & $6.3(5.3)$ & $9.5(8.2)$ & $22.2(4.3)$ \\
L-SD & 50 & $10^{-10}$ & $1.5 \times 10^{-4}$ & $51.2(3.7)$ & $60.9(4.5)$ & $69.2(5.2)$ & $364.2(0.9)$ \\
\hline
\end{tabular}

good discrimination threshold, so further developments are needed in order to use this target in a DM experiment.

In this paper we focus on two other targets: $\mathrm{CaF}_{2}$ and NaI. Fluorine-based scintillators are particularly attractive for DM searches because of the sensitivity of ${ }^{19} \mathrm{~F}$ ( $J=1 / 2,100 \%$ isotopic abundance) to SD interactions. Among them, $\mathrm{CaF}_{2}$ presents the highest light yield ${ }^{58}$ and has already been used in several DM searches as scintillator at room temperature. ${ }^{59-61}$ It was the target material of the first scintillating bolometer ever constructed, ${ }^{30}$ although in that experiment the light measurement was performed with a silicon photodiode, less sensitive than the semiconductor bolometers usually used in recent setups. ${ }^{31}$ Scintillation at low temperature has been studied for pure and europium-activated targets, resulting in good scintillation at $1 \mathrm{~K}$ specially for doped samples, ${ }^{62,63}$ although the radiopurity levels achieved in this case are usually worse.

NaI, on the other hand, is one of the most widely used scintillators for $\gamma$ spectroscopy due to its very high light yield. As mentioned above, this is the target used by DAMA/LIBRA and other proposed DM experiments looking for annual modulation. ${ }^{64,65}$ Although NaI is usually doped with $\mathrm{Tl}$ for room temperature applications, the pure material is known to scintillate better at temperatures of a few Kelvin ${ }^{66}$ (nevertheless, an increase in light yield of the Tl-doped material below $30 \mathrm{~K}$ has been recently reported ${ }^{32,67}$ ). Despite its high light yield at low temperature and intrinsic interest for DM searches, this material has not been tested yet as a bolometer due to its fragility and high hygroscopicity.

\section{Results with Bolometric Targets}

Let us now investigate the complementarity potential of scintillating bolometer targets of $\mathrm{CaF}_{2}$ and $\mathrm{NaI}$ with the Ge and Xe experiments. For both bolometric targets, we assume an energy window from 10 to $100 \mathrm{keV}$, a 5\% energy resolution and, as we have done previously for Ge and Xe, a total exposure of $300 \mathrm{~kg} \times \mathrm{yr}$ and a zero background experiment. Table 3 gives the number of recoil events for each of the bolometric targets. In the case of $\mathrm{NaI}$, three different quenching factors have been considered ( $q=0.85,1$ and 1.15). Following the same procedure of Ref. 24 , for each benchmark and target we have derived the contour plots after the combination of data from a Ge detector, a Xe detector and the corresponding bolometric target 

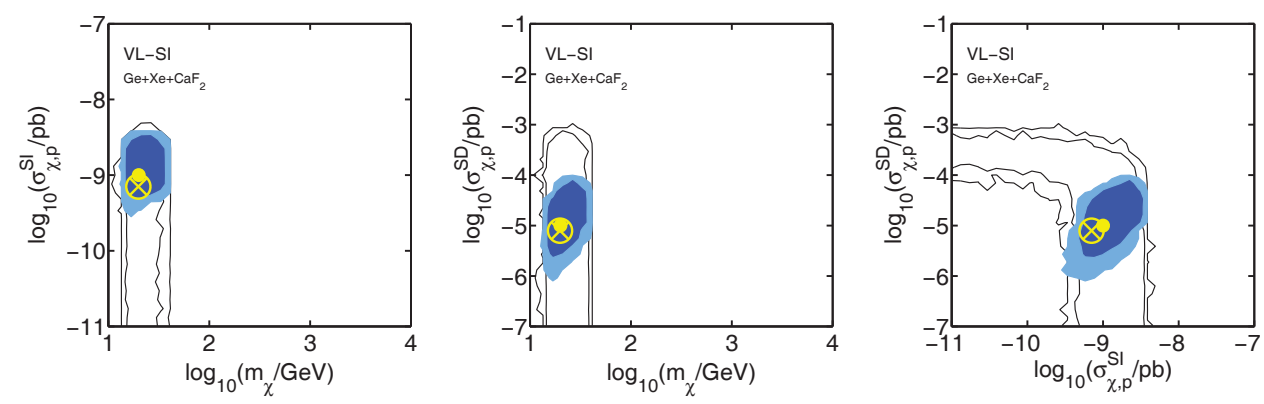

Fig. 4. (Color online) Profile likelihood contours at 68 and $99 \%$ C.L. The blue contours correspond to $\mathrm{Ge}+\mathrm{Xe}+\mathrm{CaF}_{2}$ while the empty one to only $\mathrm{Ge}+\mathrm{Xe}$. The yellow dot denotes the benchmark VL-SI and the circled cross the best fit point.

(see Figs. 4 to 7). Results are shown as blue contours, while black lines correspond to the case when only Ge and Xe are used.

In benchmark VL-SI, for which Ge and Xe exhibited a degeneracy in the $\left(\sigma^{\mathrm{SI}}, \sigma^{\mathrm{SD}}\right)$ plane, $\mathrm{CaF}_{2}$ provides a good complementarity, allowing the full reconstructions of the WIMP parameter space (see Fig. 4). This is because for this BM the $97 \%(95 \%)$ of the signal in $\mathrm{Ge}(\mathrm{Xe})$ is due to the SI component whereas in the case of $\mathrm{CaF}_{2}$, a target very sensitive to SD WIMP-nucleon interactions, the $80 \%$ of the total rate is due to the $\mathrm{SD}$ component.

The results using NaI are represented in Fig. 5, where the three rows correspond to the three different values considered for the quenching factor. As we can observe, we are able to obtain closed contours for $\sigma^{\mathrm{SI}}$, but not for $\sigma^{\mathrm{SD}}$ (see Fig. 5). The reason is that, as in the case of Ge and Xe, the signal for this target is dominated by the SI contribution (approximately $85 \%$ of the total rate). The change in the quenching factor (which can be understood as a shift in the energy window of nuclear recoils) leads to variations in the number of events due to SD and SI interactions. More importantly these do not change by the same amount, since the energy dependence of the SD and SI form factors is different. For NaI we observe that the relative contribution due to the SD term increases as the quenching factor decreases, shifting from $14 \%$ at $q=1.15$ to $17 \%$ at $q=0.85$. This implies that, for this benchmark, the complementarity with $\mathrm{Ge}$ and $\mathrm{Xe}$ is better for $q=0.85$, as we can observe in Fig. 5. The effect is clearer in the 1-D profile likelihood of the SD cross-section shown in Fig. 8. Notice also that, although the upper limit on $\sigma^{\mathrm{SD}}$ is more stringent for $q=0.85$, the derived 1-D profile likelihood is practically flat (Fig. 8 (right)) and that leads to a failure in the estimation of $\sigma^{\mathrm{SD}}$ by the best-fit point (Fig. 5).

The results for benchmark L-SD are shown in Fig. 6 for the combination of data from Xe, Ge, and $\mathrm{CaF}_{2}$, and in Fig. 7 for NaI. As we can see in Tables 2 and 3 , in this benchmark the WIMP interactions are dominated by the SD contribution for all the targets. Consequently, the degeneracy in the $\left(\sigma^{\mathrm{SI}}, \sigma^{\mathrm{SD}}\right)$ plane is not completely removed, although the contours are substantially reduced with respect 

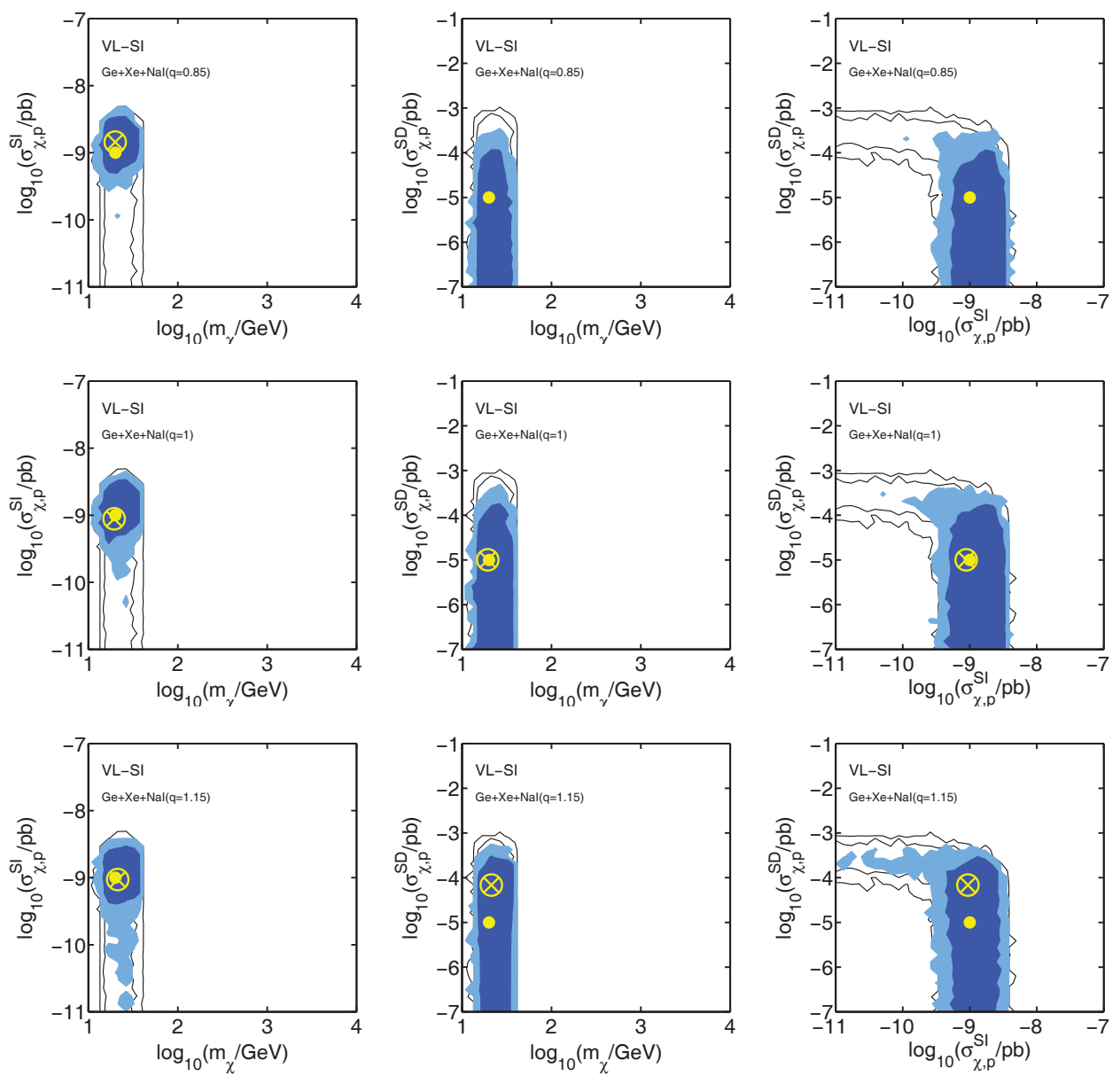

Fig. 5. (Color online) The same as Fig. 4 but for the combination $\mathrm{Ge}+\mathrm{Xe}+\mathrm{NaI}$. From top to bottom the quenching factor for $\mathrm{NaI}$ is $0.85,1$ and 1.15 , respectively.
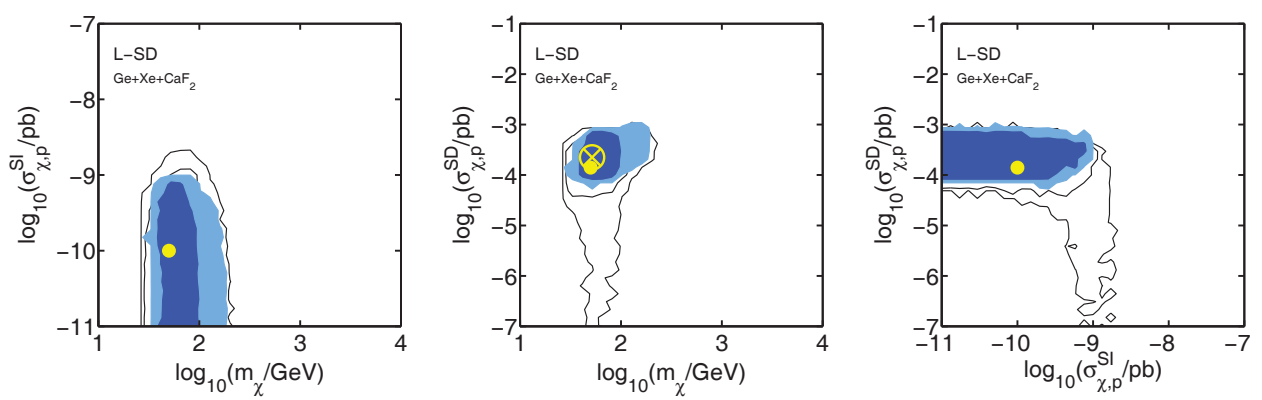

Fig. 6. (Color online) The same as Fig. 4 but for the L-SD benchmark. 

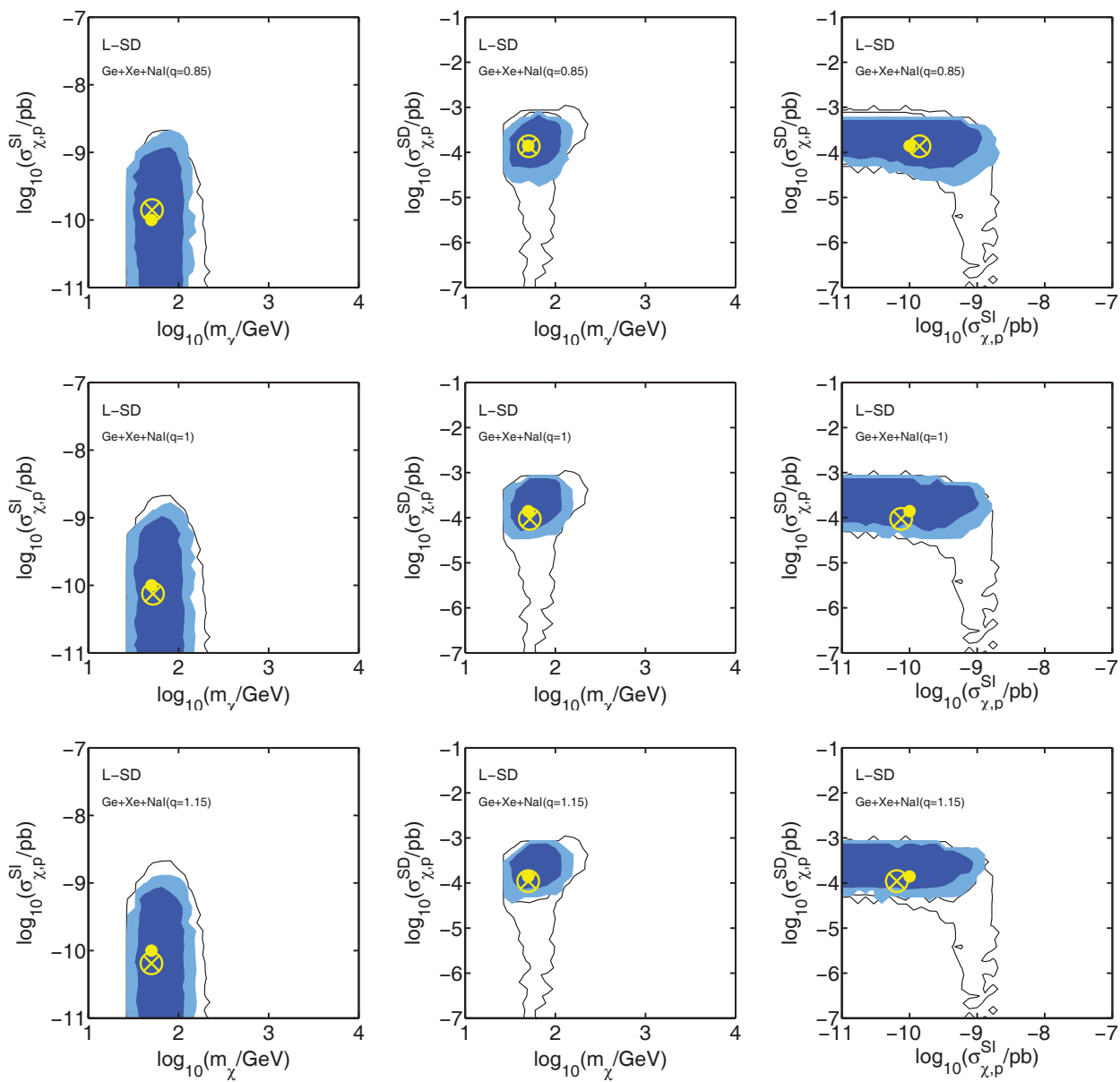

Fig. 7. (Color online) The same as Fig. 5 but for the L-SD benchmark.
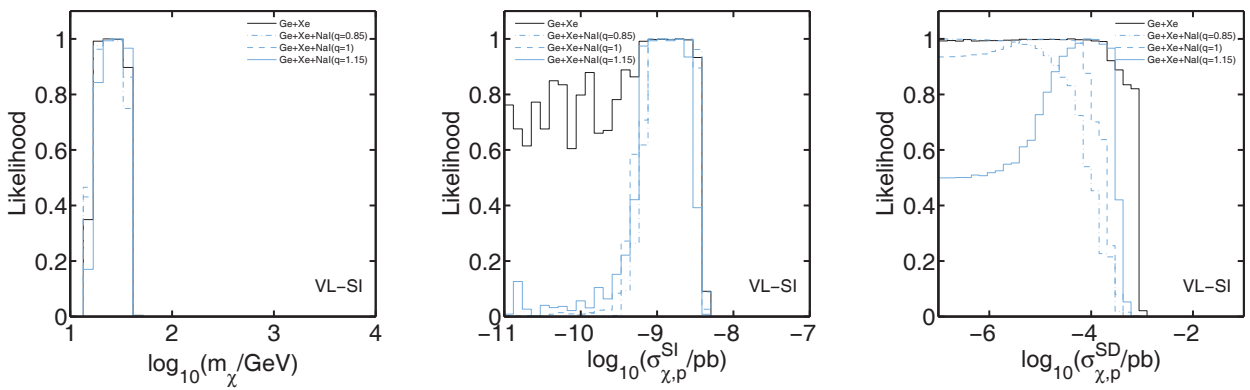

Fig. 8. 1-D profile likelihood plots for $\mathrm{Ge}+\mathrm{Xe}$ and $\mathrm{Ge}+\mathrm{Xe}+\mathrm{NaI}$, considering three different thermal quenching values $(q=0.85,1,1.15)$ for benchmark VL-SI. 

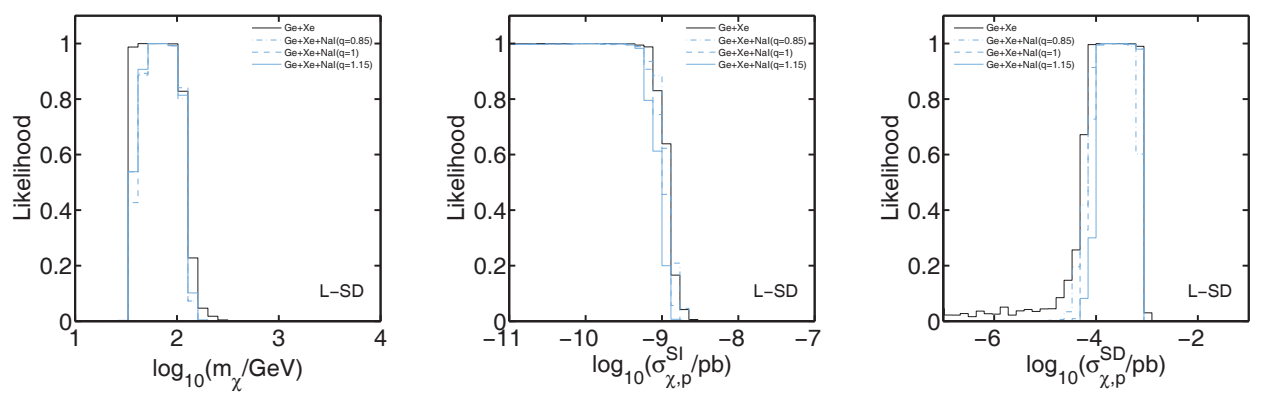

Fig. 9. The same as Fig. 8 for the L-SD benchmark.

to the case with Ge and Xe alone. In particular, closed contours appear for $\sigma^{\mathrm{SD}}$ around the nominal value with both $\mathrm{CaF}_{2}$ and $\mathrm{NaI}$, but only an upper bound for $\sigma^{\mathrm{SI}}$ is obtained. In this benchmark the effect of the quenching factor is quite imperceptible (see also Fig. 9) because the relative contribution of the SD term is practically the same (approximately $7.5 \%$ ) for the three values of $q$.

\section{Conclusions}

Following the work done in Ref. 24, where we investigated the determination of WIMP parameters $\left(m_{\chi}, \sigma^{\mathrm{SI}}, \sigma^{\mathrm{SD}}\right)$ from a hypothetical direct DM detection with multiple targets, in this paper we have extended the analysis to consider the effect of lower thresholds in Ge and Xe targets, as well as the complementarity potential of two new bolometric targets: $\mathrm{CaF}_{2}$ and $\mathrm{NaI}$.

We first considered the combination of data from Ge and Xe targets, for both of which we assumed a low energy threshold of $3 \mathrm{keV}$ to account for recent or projected experimental improvements. We studied two benchmark scenarios, featuring a very light WIMP $\left(m_{\chi}=20 \mathrm{GeV}, \sigma^{\mathrm{SI}}=10^{-9} \mathrm{pb}, \sigma^{\mathrm{SD}}=10^{-5} \mathrm{pb}\right)$ in which SI contribution dominates the detection rate in both Ge and $\mathrm{Xe}$, and a light WIMP $\left(m_{\chi}=50 \mathrm{GeV}, \sigma^{\mathrm{SI}}=10^{-10} \mathrm{pb}, \sigma^{\mathrm{SD}}=1.5 \times 10^{-4} \mathrm{pb}\right)$ in which the SD contribution dominates. Although the combination of data from both targets allows a significant improvement in the reconstruction of DM parameters, a degeneracy in the $\left(\sigma^{\mathrm{SI}}, \sigma^{\mathrm{SD}}\right)$ plane usually remains in the points in the parameter space where both targets have similar SI/SD ratios.

Scintillating bolometers, with very good energy threshold and resolution and particle discrimination capability, provide a wide choice of absorber materials that allows to select interesting targets form the point of view of its complementarity with other experiments. In Ref. 24 we studied how certain bolometric targets $\left(\mathrm{CaWO}_{4}, \mathrm{Al}_{2} \mathrm{O}_{3}\right.$ and $\left.\mathrm{LiF}\right)$ could provide complementary information to data from Ge or Xe based experiments. In this work we have extended the analysis to other two scintillating targets $\left(\mathrm{CaF}_{2}\right.$ and $\left.\mathrm{NaI}\right)$, and considered also the effect of an uncertainty in the thermal quenching factor of $\pm 15 \%$. Both targets are sensitive to the SD component of the WIMP-nucleus interaction (particularly $\mathrm{CaF}_{2}$ thanks to the presence of ${ }^{19} \mathrm{~F}$ ). 
We have shown how the inclusion of one of these targets together with Ge and Xe can help breaking the degeneracy in the $\left(\sigma^{\mathrm{SI}}, \sigma^{\mathrm{SD}}\right)$ plane. In particular, in the points of the parameter space for which the rate in Ge and Xe is dominated by the SI contribution and the rate in $\mathrm{CaF}_{2}$ is mostly $\mathrm{SD}$, the three DM parameters can be reconstructed. In other examples, although the degeneracy cannot completely removed, at least one of the components of the WIMP-nucleus scattering crosssection can be determined.

We have also shown how a small uncertainty in the thermal quenching factor can modify noticeably the parameter reconstruction.

\section{Acknowledgments}

D. G. Cerdeño is supported by the Ramón y Cajal program of the Spanish MICINN. M. Fornasa is supported by a Leverhulme Trust grant. M. Peiró is supported by a MultiDark Scholarship. Y. Ortigoza is supported by a MultiDark Fellowship. We also thank the support of the Consolider-Ingenio 2010 programme under grant MULTIDARK CSD2009-00064, the Spanish MICINN under Grant No. FPA201234694, the Spanish MINECO "Centro de excelencia Severo Ochoa Program" under Grant No. SEV-2012-0249, the Community of Madrid under Grant No. HEPHACOS S2009/ESP-1473, the Spanish and the European Regional Development Fund MINECO-FEDER under grant FPA2011-23749, the Government of Aragón, and the European Union under the Marie Curie-ITN Program No. PITN-GA-2009-237920. We gratefully acknowledge the access to the High Performance Computing Facility of the University of Nottingham and to the Computing Facilities at the Instituto de Física Teórica.

\section{References}

1. M. W. Goodman and E. Witten, Phys. Rev. D 31, 3059 (1985).

2. R. Bernabei et al., Riv. Nuovo Cimento 26, 1 (2003).

3. R. Bernabei et al., Eur. Phys. J. C 56, 333 (2008).

4. R. Bernabei et al., Eur. Phys. J. C 73, 2648 (2013).

5. CoGeNT Collab. (C. Aalseth et al.), arXiv:1401.3295 [astro-ph.CO].

6. C. Aalseth et al., arXiv:1401.6234 [astro-ph.CO].

7. C. E. Aalseth et al., Phys. Rev. Lett. 107, 141301 (2011).

8. G. Angloher et al., Eur. Phys. J. C 72, 1971 (2012).

9. CDMS Collab. (R. Agnese et al.), Phys. Rev. Lett. 111, 251301 (2013).

10. J. Angle et al., Phys. Rev. Lett. 107, 051301 (2011).

11. E. Aprile et al., Phys. Rev. Lett. 109, 181301 (2012).

12. LUX Collab. (D. S. Akerib et al.), arXiv:1310.8214 [astro-ph.CO].

13. Z. Ahmed et al., Science 327, 1619 (2010).

14. E. Armengaud et al., Phys. Lett. B 702, 329 (2011).

15. Z. Ahmed et al., Phys. Rev. D 84, 011102 (2011).

16. S. C. Kim et al., Phys. Rev. Lett. 108, 181391 (2012).

17. S. Archambault et al., Phys. Lett. B 711, 153 (2012).

18. M. Felizardo et al., Phys. Rev. Lett. 108, 201302 (2012). 
19. E. Behnke et al., Phys. Rev. Lett. 106, 021303 (2011).

20. SuperCDMSSoudan Collab. (R. Agnese et al.), Phys. Rev. Lett. 112, 041302 (2014), arXiv:1309.3259 [physics.ins-det].

21. SuperCDMS Collab. (R. Agnese et al.), arXiv:1402.7137 [hep-ex].

22. G. Bertone, D. G. Cerdeño, J. I. Collar and B. Odom, Phys. Rev. Lett. 99, 151301 (2007).

23. M. Pato et al., Phys. Rev. D 83, 083505 (2011).

24. D. Cerdeño et al., J. Cosmol. Astropart. Phys. 07, 028 (2013).

25. J. Sander et al., AIP Conf. Proc. 1534, 129 (2012).

26. XENON1T Collab. (E. Aprile et al.), arXiv:1206.6288 [astro-ph.IM].

27. N. Coron et al., PoS (IDM2010) 054 (2010).

28. H. Kraus et al., PoS (IDM2010) 109 (2011).

29. CDMS-II Collab. (Z. Ahmed et al.), Phys. Rev. Lett. 106, 131302 (2011), arXiv:1011.2482 [astro-ph.CO].

30. A. Alessandrello et al., Nucl. Phys. B (Proc. Suppl.) 28, 233 (1992).

31. C. Bobin et al., Nucl. Instrum. Methods A 386, 453 (1997).

32. N. Coron et al., Astropart. Phys. 47, 31 (2013).

33. P. F. Smith and J. D. Lewin, Phys. Rep. 187, 203 (1990).

34. J. D. Lewin and P. F. Smith, Astropart. Phys. 6, 87 (1996).

35. D. G. Cerdeño and A. M. Green, arXiv:1002.1912 [astro-ph.CO].

36. A. H. G. Peter, V. Gluscevic, A. M. Green, B. J. Kavanagh and S. K. Lee, arXiv:1310.7039 [astro-ph.CO].

37. F. Feroz, M. P. Hobson and M. Bridges, Mon. Not. R. Astron. Soc. 398, 1601 (2009).

38. M. Lisanti, L. E. Strigari, J. G. Wacker and R. H. Wechsler, Phys. Rev. D 83, 023519 (2011), arXiv:1010.4300 [astro-ph.CO].

39. D. Cerdeño, M. Fornasa, J. Huh and M. Peiro, Phys. Rev. D 87, 023512 (2013).

40. P. Klos, J. Menéndez, D. Gazit and A. Schwenk, Phys. Rev. D 88, 083516 (2013), arXiv:1304.7684 [nucl-th].

41. G. Belanger, F. Boudjema, A. Pukhov and A. Semenov, Comput. Phys. Commun. 180, 747 (2009), arXiv:0803.2360 [hep-ph].

42. M. T. Ressell et al., Phys. Rev. D 48, 5519 (1993).

43. V. Dimitrov, J. Engel and S. Pittel, Phys. Rev. D 51, 291 (1995), arXiv:hep$\mathrm{ph} / 9408246$.

44. M. Ressell and D. Dean, Phys. Rev. C 56, 535 (1997), arXiv:hep-ph/9702290.

45. P. Divari, T. Kosmas, J. Vergados and L. Skouras, Phys. Rev. C 61, 054612 (2000).

46. N. Coron et al., Phys. Lett. B 659, 113 (2008).

47. D. Malling et al., arXiv:1110.0103 [astro-ph.IM].

48. DARWIN Consortium Collab. (L. Baudis), J. Phys. Conf. Ser. 375, 012028 (2012), arXiv:1201.2402 [astro-ph.IM].

49. C. Isaila et al., Phys. Lett. B 716, 160 (2012).

50. J. Beeman et al., Nucl. Instrum. Methods A 709, 22 (2013).

51. J. Amaré et al., J. Phys. Conf. Ser. 39, 133 (2006).

52. S. Cebrián et al., Phys. Lett. B 563, 48 (2003).

53. J. Amaré et al., Appl. Phys. Lett. 87, 264102 (2005).

54. A. Calleja et al., J. Low Temp. Phys. 151, 848 (2008).

55. F. Proebst et al., Nucl. Phys. B (Proc. Suppl.) 110, 67 (2002).

56. M. Martínez et al., J. Phys. Conf. Ser. 375, 012026 (2012).

57. N. Coron et al., J. Phys. Conf. Ser. 203, 012139 (2010).

58. S. K. Kim, H. J. Kim and Y. D. Kim, New J. Phys. 12, 075003 (2010). 
59. I. Ogawa et al., Nucl. Phys. A 663-664, 869c (2000).

60. R. Bernabei et al., WIMP search by the DAMA experiment at Gran Sasso, in Dark Matter in Astro- and Particle Physics, eds. H. Klapdor-Kleingrothaus and R. Viollier (Springer, Berlin, Heidelberg, 2002), pp. 459-472.

61. Y. Shimizu, M. Minowa, W. Suganuma and Y. Inoue, Phys. Lett. B 633, 195 (2006).

62. N. Coron et al., Nucl. Instrum. Methods A 520, 159 (2004) [Proceedings of the 10th International Workshop on Low Temperature Detectors].

63. V. Mikhailik, H. Kraus, J. Imber and D. Wahl, Nucl. Instrum. Methods A 566, 522 (2006).

64. J. Amaré et al., J. Phys. Conf. Ser. 375, 012026 (2012).

65. J. Cherwinka et al., Astropart. Phys. 35, 749 (2012), arXiv:1106.1156 [astro-ph.HE].

66. W. J. Van Sciver and L. Bogart, IRE Trans. Nucl. Sci. 5, 90 (1958).

67. C. Sailer, B. Lubsandozhiev, C. Strandhagen and J. Jochum, Eur. Phys. J. C 72, 2061 (2012), arXiv:1203.1172 [physics.ins-det]. 